\title{
Valores económicos relativos para caracteres de finura en fibra de alpacas de la Región Puno
}

\author{
Relative economic values for fineness traits in alpaca fibre of the Puno Region \\ Edgar Apaza Zuñiga ${ }^{1,3}$, Samuel Cazorla Chambi ${ }^{2}$, Cesar Condori Carbajal'2, \\ Fredy Ricardo Arpasi Meléndez ${ }^{2}$, Isabel Tumi Figueroa ${ }^{2}$, William Yana Viveros ${ }^{2}$, \\ Jesús Esteban Quispe Coaquira ${ }^{1}$
}

\section{RESUMEN}

El estudio tuvo como objetivo determinar pesos económicos relativos para 10 caracteres relacionados a la calidad de la fibra en alpacas Huacaya, a través del Recíproco de la desviación estándar fenotípica del carácter y la Regresión de los caracteres sobre el benéfico económico. Se utilizaron 22543 datos por carácter, determinados en muestras de vellón de alpacas de la Región Puno. Los pesos económicos obtenidos por el primer procedimiento asumen diferentes valores, los que son inversamente proporcionales al valor de la desviación estándar fenotípica del carácter, y cuantifican el número de veces que la variancia contiene a la desviación estándar fenotípica pertinente. Los obtenidos por el método de regresión asumen también valores diferentes (positivos y negativos), que no necesariamente orientan la tendencia, pero cuantifican el cambio en la pendiente a un cambio en una unidad promedio del fenotipo. Los valores de pesos económicos no son similarmente proporcionales por ambos métodos. Los pesos económicos obtenidos son los más apropiados para las características relacionadas con la finura de la fibra en alpacas, pero no necesariamente considerados como criterio de selección.

Palabras clave: objetivo de cría, valor de cría agregado, peso económico

\footnotetext{
${ }^{1}$ Facultad de Medicina Veterinaria y Zootecnia, Universidad Nacional del Altiplano, Puno, Perú

${ }^{2}$ Gobierno Regional Puno, Proyecto Especial Camélidos Sudamericanos, Puno, Perú

${ }^{3}$ E-mail: edapazaz@gmail.com
}

Recibido: 5 de enero de 2021

Aceptado para publicación: 29 de mayo de 2021

Publicado: 24 de agosto de 2021

CLos autores. Este artículo es publicado por la Rev Inv Vet Perú de la Facultad de Medicina Veterinaria, Universidad Nacional Mayor de San Marcos. Este es un artículo de acceso abierto, distribuido bajo los términos de la licencia Creative Commons Atribución 4.0 Internacional (CC BY 4.0) [https:// creativecommons.org/licenses/by/4.0/deed.es] que permite el uso, distribución y reproducción en cualquier medio, siempre que la obra original sea debidamente citada de su fuente original 
The aim of this of the study was to determine relative economic weights for 10 traits related to fibre quality in Huacaya alpacas, through the Reciprocal of the phenotypic standard deviation of the trait and the Regression of the traits on the economic benefit. In total, 22543 data per character were used, determined in samples of fleece from alpacas from the Puno Region. The economic weights obtained by the first procedure assume different values, which are inversely proportional to the value of the phenotypic standard deviation of the trait and quantify the number of times that the variance contains the relevant phenotypic standard deviation. Those obtained by the regression method also assume different values (positive and negative), which do not necessarily guide the trend, but quantify the change in the slope to a change in an average unit of the phenotype. The economic weight values are not similarly proportional by both methods. The economic weights obtained are the most appropriate for the characteristics related to the fineness of the fibre in alpacas, but not necessarily considered as selection criteria.

Key words: breeding objective, value of breeding aggregate, economic weight

\section{INTRODUCCIÓN}

El mejoramiento genético animal es un conjunto de procesos selectivos, cuyo propósito es incrementar la frecuencia de genes deseables con la consecuente disminución de los no deseables para un carácter en la población, lo que se logra con la selección. Existen varios métodos de selección donde cada uno especifica la magnitud de la ganancia genética por generación de selección (Piedrafita, 1995). Uno de ellos es la selección por caracteres múltiples, y dentro de ella se concibe a los índices de selección; método que puede ser utilizado en la mejora genética de caracteres productivos en alpacas, pero que circunstancialmente no es utilizada por el compromiso de los años que implica la implementación de estos programas de mejora genética.

En la construcción de un índice de selección es imprescindible determinar los ponderados, pesos o valores económicos que se le atribuye a cada carácter que constituye el objetivo de cría del Mérito Genético Total; valores que reflejan la importancia económi- ca que se le asigna a cada carácter que constituye el objetivo de cría. El Peso Económico (PE) expresa el cambio en la ganancia del rebaño como consecuencia de una unidad de cambio en el mérito genético del carácter considerado (Charfeddine, 2000; Ramón et al., 2005) y responde a las condiciones de mercado del periodo en el que se desarrolla el plan de mejora; es decir, proviene de un análisis de tendencias de mercado (Mueller, 1985). Así, derivar valores económicos para caracteres objetivo de selección implica el primer paso para mejorar el criterio de selección (Rewe et al., 2006; Kahi y Nitter, 2004; Ponzoni y Newman, 1989), tarea que a menudo es difícil (Romero, 2000).

Para estimar el PE para características objetivo de selección se dispone de métodos objetivos y no objetivos (Groen et al., 1997). En los primeros resalta el modelado, expresado en una ecuación o un juego de ecuaciones que representan a las características del sistema de producción, y que constituyen una Función beneficio o Modelo bioeconómico (Harris y Freeman, 1993; Weigel et al., 1995); dentro de ellos existen dos acercamientos de análisis: i) el positivo o 
evaluación de datos y ii) el normativo o simulación de datos. En tanto, los métodos no objetivos establecen índices de ganancia genética deseada o restringida (Brascamp, 1978). Para el efecto, los PE de los caracteres son calculados asignando la ganancia genética requerida para cada rasgo (Elsen et al., 1986; Groen, 1989) o son fijados por una aproximación «ad-hoc»; en este último caso, los PE de los caracteres son definidos por una decisión subjetiva de los criadores. En este contexto, la Función beneficio representa la relación entre la producción animal por rasgos económicamente importantes y el beneficio o resultado económico en el nivel de la granja (Bourdon, 1998); procedimiento que describe el cambio en el retorno económico neto como una función de una serie de parámetros físicos, biológicos y económicos (Gibson et al., 1992).

El acercamiento positivo incluye al análisis de regresión, que establece la relación entre el beneficio del sistema y la de los valores de cría de los animales para el (los) carácter(es) evaluado(s); sin embargo, esta aproximación requiere de la necesidad de una gran cantidad de datos del sistema de producción evaluada, y los PE son calculados sobre la base de los niveles y precios alcanzados en el pasado. Paradójicamente, los ganaderos se orientan al futuro, razón por la cual la aproximación normativa es preferida por muchos autores (Krupová et al., 2008). Consecuentemente, la complejidad de los sistemas de producción ganadera requiere asumir escenarios de producción a futuro, que expresen la mejora genética que se inicia, y la predicción de los $\mathrm{PE}$ de los productos a obtener, así como los costos de producción correspondientes, todo lo cual hace que la estimación de los PE sea un proceso complejo.

Varios son los reportes de investigación científica que refieren el uso de diferentes procedimientos para determinar el PE. Atil (2006) compara diferentes índices de selección para la mejora genética de caracteres en producción de leche en vacunos Holstein Friesian, aplicando los recíprocos de las desviaciones fenotípicas de cada carácter; Kluyts et al. (2007) utilizan la derivada parcial de la Función beneficio en vacunos Simmental para caracteres de crecimiento y calidad de carcasa; Legarra et al. (2007) estiman PE a través de una función beneficio en ovinos de leche Manchega y Latxa para caracteres de producción de leche, fertilidad, prolificidad y vida productiva.

Del mismo modo, otros autores como Fernández y Alenda (1999), Parna et al. (2002), Yáñez et al. (2006), Krupová et al. (2008) y Chen et al. (2009) estiman PE para caracteres funcionales y de producción en vacunos y ovinos a través de modelos bioeconómicos, lo cual evidencia la amplia variedad de métodos para la determinación de PE. Sin embargo, no es posible proponer la «mejor» metodología en la derivación de los PE, pues la «mejor» dependerá de las características y circunstancias consideradas en determinado sistema de producción (Parna et al., 2002). El mejor método teórico no necesariamente es el más práctico a implemen$\operatorname{tar}$ (Groen et al., 1997), por lo que, en la derivación de PE es importante estar conscientes de que la mejora genética es un desarrollo tecnológico.

Ante la ausencia de información científica de estos parámetros en la alpaca, se planteó la determinación de los PE para características relacionadas a la finura de la fibra en alpacas Huacaya de la Región Puno, planteando el objetivo de determinar PE para las características: Media de diámetros de la fibra (DMF), Desviación estándar del diámetro de fibra (DE), Coeficiente de variabilidad del diámetro de fibra (CDV), Coarse Edge Micron (CEM), Porcentaje de fibras menores a 15 micras (FINO15), Factor de confort de la fibra (FDEC), Finura de hilado (FDEH), Largo de la mecha (LMECHA), Finest point from the tip (FPFT) e Índice de curvatura (CRV), a través del recíproco de las desviación típica fenotípica y regresión múltiple, 
asumiendo que el objetivo de selección es el mismo que el criterio de selección. Se asume que estos parámetros posibiliten la construcción de índices de selección genética para el agregado genético total en programas de mejora genética de la alpaca.

\section{Materiales y Métodos}

\section{Lugar del Estudio}

Se utilizaron 22543 muestras de vellón, obtenidos de alpacas Huacaya de diferentes edades y sexo, cuya base alimenticia fueron pastos naturales de praderas altoandinas, bajo un sistema de explotación extensiva. Los animales eran procedentes de 12 provincias del departamento de Puno. Se consideraron 55 distritos y 362 comunidades, todos distribuidos a lo largo de los dos sectores de la Zona Agroecológica Puna: Puna Seca (PS) y Puna Húmeda (PH) de la Región Puno (ALTPNUD, 2001).

\section{Análisis de Fibra}

Las muestras de fibra fueron analizadas en el Laboratorio de Fibras del Proyecto Especial Camélidos Sudamericanos (PECSA) del Gobierno Regional Puno, utilizando un equipo OFDA 2000, instrumento diseñado y propuesto el Interactive Wool Group (IWG) para la medición de diferentes características en muestra de fibra sucia, con rapidez y precisión (Elvira, 2014). Se analizaron las variables que evidencian características físicas y textiles de la fibra como Media de diámetros de la fibra (DMF), Desviación estándar del diámetro de fibras (DE), Coeficiente de variabilidad del diámetro de fibras (CDV), Coarse Edge Micron (CEM), Porcentaje de fibras menores a 15 micras (FINO15), Factor de confort de la fibra (FDEC), Finura de hilado (FDEH), Largo de la mecha (LMECHA), Finest point from the tip (FPFT) e Índice de curvatura (CRV).

\section{Análisis Estadístico}

Uno de los procedimientos metodológicos utilizados para la determinación de los PE $\left(a_{i}\right)$ fue planteado por Falconer y Mackay (1996) y Atil (2006), quienes sostienen que los PE son asignados a una desviación estándar del cambio de cada carácter, determinados específicamente a través de los recíprocos de la desviación estándar fenotípica: $\sigma_{\mathrm{p}} / \sigma_{\mathrm{p}}^{2}$. En el presente estudio se sistematizó y procesó a través de la hoja electrónica Excel del Microsoft. El segundo procedimiento fue expuesto por James (1982) y sustentado por MacNieil et al. (1997) y Pearson y Miller (1981), quienes indican que los PE pueden ser derivados por regresión parcial de un simple carácter sobre el beneficio (profit) por animal. En este estudio se empleó el procedimiento de la regresión múltiple de los caracteres sobre la variable profit para determinar PE relativos. Para ello, se utilizó el software SAS v. 9.2, implementando el siguiente modelo estadístico: $y_{i}=\beta_{0}+\beta_{I} X_{1}+\beta_{2} X_{2}+$ $\ldots+\beta_{10} X_{10}$, en donde $w_{i}$ es una combinación lineal las variables independientes $\left(X_{1}\right)$ cada una de ellas ponderada por un coeficiente de regresión $\left(\beta_{j}\right)$, a través de un programa específico (Duangjinda, 2007).

La variable beneficio $y_{i}$ (profit) solo consideró el valor económico de la libra de fibra sucia pagada al productor. A este precio se le añadió un monto económico definido por la característica diámetro de fibra hasta un máximo de $\mathrm{S} / .2 .50$ [ $\$ 1.00=\mathrm{S} / .3 .357$, diciembre de 2016] para aquellas fibras cuyo promedio fue menor a $23 \mu \mathrm{m}$, clasificadas como «baby». Este monto fue disminuyendo hasta $\mathrm{S} / .0 .0$ soles para aquellas cuyos promedios fueron de $31.6 \mu \mathrm{m}$ a más, en concordancia a la Tabla de clasificación establecida por la Norma Técnica Peruana 231.302.2014 (INDECOPI, 2014). 
Cuadro 1. Valores económicos relativos para características de la finura de la fibra en alpacas Huacaya por el método del reciproco de la desviación estándar fenotípica $(\mathrm{n}=22543)$

\begin{tabular}{|c|c|c|c|}
\hline Variable & $\sigma_{P}$ & $\sigma_{P}^{2}$ & $\begin{array}{c}\text { Peso } \\
\text { económico }\end{array}$ \\
\hline Media del diámetro de fibra (MDF) & 3.3904 & 11.4949 & 0.2949 \\
\hline $\begin{array}{l}\text { Desviación Estándar del diámetro de } \\
\text { fibra (DE) }\end{array}$ & 0.9998 & 0.9995 & 1.0002 \\
\hline $\begin{array}{l}\text { Coeficiente de variabilidad del } \\
\text { diámetro de fibra (CDV) }\end{array}$ & 3.3577 & 11.2744 & 0.2978 \\
\hline Coarse edge measurement (CEM) & 1.8044 & 3.2559 & 0.5542 \\
\hline $\begin{array}{l}\text { Porcentaje de fibras menor a } 15 \mu \mathrm{m} \\
\text { (FINO15) }\end{array}$ & 11.5213 & 132.7411 & 0.0868 \\
\hline Factor de confort (FDEC) & 9.1879 & 84.4166 & 0.1088 \\
\hline Finura de hilado (FDEH) & 3.3583 & 11.2781 & 0.2978 \\
\hline Longitud de mecha (LMECHA) & 28.0435 & 786.4406 & 0.0357 \\
\hline Finest point from the tip (FPFT) & 30.2800 & 916.8790 & 0.0330 \\
\hline Índice de curvatura (CRV) & 9.3265 & 86.9833 & 0.1072 \\
\hline
\end{tabular}

\section{Resultados}

\section{Método del Recíproco de la Desviación Estándar Fenotípica}

Los resultados de los PE para las características MDF, DE, CDV, CEM, FINO15, FDEC, FDEH, LMECHA, FPFT y CRV de la fibra del vellón de alpacas Huacaya de la Región Puno determinados por el método del reciproco de la desviación estándar fenotípica se presentan en el Cuadro 1.

Se observa que asumen valores diferentes, correspondiendo valores menores a mayores valores de variancia fenotípica del carácter. Un valor anormal corresponde al carácter: Desviación Estándar del diámetro de la fibra (DE), cuya desviación estándar es un valor próximo a la unidad (1), lo que implica que la variancia y el valor del PE sean tam- bién iguales a uno, no coherentes con la definición de PE. Sin embargo, valores similares se obtuvieron para los caracteres MDF, CDV y FDEH, dada su estrecha relación fenotípica. Valores relativamente menores se obtuvieron para en el resto de los caracteres.

El método resulta viable y adecuado para los caracteres en estudio en esta especie animal, dentro del variado conjunto de procedimientos que requieren de una cantidad de datos e información socioeconómica que posibilitan la determinación de PE reales. Su magnitud en términos numéricos evidencia la cantidad de veces que la desviación estándar fenotípica del carácter está contenida en la variancia de la misma, e implican la magnitud en la que se espera se incremente el beneficio económico al incrementarse en una unidad el carácter en mención, si todos ellos estuvieran considerados en el agregado genotípico total. 
Cuadro 2. Valores económicos relativos para características de la finura de la fibra en alpacas Huacaya por el método de regresión $(n=22543)$

\begin{tabular}{lccccc}
\hline & \multicolumn{4}{c}{ Estimadores de parámetros } \\
\cline { 2 - 6 } Variable & GL & $\begin{array}{c}\text { Estimador del } \\
\text { parámetro }\end{array}$ & $\begin{array}{c}\text { Error } \\
\text { estándar }\end{array}$ & Valor $\mathrm{t}$ & $\mathrm{p}>|\mathrm{t}|$ \\
\hline $\begin{array}{l}\text { Media del diámetro de } \\
\text { fibra (MDF) }\end{array}$ & 1 & -0.68842 & 0.06345 & -10.85 & $<.0001$ \\
$\begin{array}{l}\text { Desviación estándar del } \\
\text { diámetro de fibra (DE) }\end{array}$ & 1 & 1.01383 & 0.08693 & 11.66 & $<.0001$ \\
$\begin{array}{l}\text { Coeficiente de } \\
\text { variabilidad del diámetro } \\
\text { de fibra (CDV) }\end{array}$ & 1 & -0.39766 & 0.01071 & -37.13 & $<.0001$ \\
$\begin{array}{l}\text { Coarse edge } \\
\text { measurement (CEM) }\end{array}$ & 1 & -0.04770 & 0.00899 & -5.30 & $<.0001$ \\
$\begin{array}{l}\text { Porcentaje de fibras }<15 \\
\mu m \text { (FINO15) }\end{array}$ & 1 & 0.12534 & 0.00114 & 109.68 & $<.0001$ \\
$\begin{array}{l}\text { Factor de confort } \\
\text { (FDEC) }\end{array}$ & 1 & 0.05534 & 0.00147 & 37.58 & $<.0001$ \\
$\begin{array}{l}\text { Finura de hilado } \\
\text { (FDEH) }\end{array}$ & 1 & 0.70863 & 0.08025 & 8.83 & $<.0001$ \\
$\begin{array}{l}\text { Longitud de mecha } \\
\text { (LMECHA) }\end{array}$ & 1 & -000046246 & 0.00025247 & -1.83 & 0.0670 \\
$\begin{array}{l}\text { Finest point from the tip } \\
\text { (FPFT) }\end{array}$ & 1 & -0.00020195 & 0.00026358 & -0.77 & 0.4436 \\
$\begin{array}{l}\text { Índice de curvatura } \\
\text { (CRV) }\end{array}$ & 1 & 0.00214 & 0.00085011 & 2.52 & 0.0118 \\
\hline
\end{tabular}

\section{Método de Regresión}

Los resultados de los PE, hallados por análisis de regresión para las características MDF, DE, CDV, CEM, FINO15, FDEC, FDEH, LMECHA, FPFT y CRV de la fibra del vellón se presentan en el Cuadro 2.

Los PE nominados como Estimados del parámetro para las características en referencia, a diferencia del método anterior, asumen valores positivos y negativos. Entre los negativos resaltan los pertinentes a las ca- racterísticas MDF y CDV, el primero refiere que a menor diámetro de la fibra le correspondería un mayor ingreso económico, razonamiento similar sería aplicable al CVD. Por otro lado, los otros PE negativos no tienen relevancia por su valor cercano a cero, a pesar de tener alta significancia estadística $(p<0.01)$. Entre los positivos resaltan las características FDEH y FINO15, mientras que el resto no son relevantes, aunque son también altamente significantes. Un caso atípico presenta la característica DE que asumió un valor unitario. 
Los valores de PE determinados por ambos procedimientos metodológicos no guardan proporcionalidad con la magnitud de sus resultados. El significado de estos es igual a los considerados en el proceso anterior.

\section{Discusión}

Derivar valores económicos requiere de correctas bases teóricas, metodología propia, aspectos económicos y sociales, así como supuestos apropiados de las circunstancias de la futura producción (Charfeddine, 2000), además, información detallada sobre el sistema de producción actual e histórica; condiciones que no se satisfacen para la producción de fibra en alpacas, como consecuencia de las características del sistema de producción, mayormente para caracteres que son determinados con ayuda de equipos y software especializados. El sistema de crianza de las alpacas presenta notables diferencias del resto de la ganadería nacional debido a su peculiar ubicación altitudinal, tamaño del predio y rebaño, propósito de crianza y grado de organización (Quispe et al., 2016), más aún a nivel de pequeña y mediana ganadería, en la que la organización productiva es débil y cuya producción está orientada al mercado regional. Estas circunstancias impiden aplicar métodos más precisos en la determinación de PE reales en alpacas, por lo que los métodos utilizados son los que mejor se ajustan a las condiciones de la explotación de la ganadería alpacuna en el Altiplano peruano.

Así mismo, se debe considerar que la determinación de los PE en algunas ocasiones está orientada específicamente para la construcción de índices de selección (Gibson et al., 1992; Saveli et al., 2003; Kluyts et al., 2007; Chen et al., 2009) o para definir criterios de selección a través de su importancia económica (Rabelo da Fonseca et al., 2000; Yáñez et al., 2006; Krupová, et al., 2008). En tanto, el propósito en el presente estudio fue viabilizar la construcción de un índice del agregado genético total, asumiendo el supues- to de que todos los caracteres son considerados como constituyentes del Objetivo de Selección, aplicable a un programa de mejora genética para características de la fibra en poblaciones de alpacas a nivel de pequeña a mediana ganadería, propias de la Región Puno.

La decisión para el uso de los procedimientos metodológicos en la determinación de los PE relativos para caracteres relacionados a la calidad de la fibra en el presente estudio queda justificada por la existencia de una gran variedad de métodos para la determinación de PE. Bobner (1994) y Krupová et al. (2008) indican procedimientos utilizando funciones que representan las características de un sistema productivo, e incluyen al análisis de regresión para establecer la relación entre el beneficio del sistema de producción y los valores de cría de los animales para los caracteres evaluados. Ante esto, se eligieron los métodos recíproco de la desviación estándar fenotípica y la regresión múltiple, por ser los que mejor se adecuan a los caracteres considerados en el presente estudio, dada la complejidad informativa y operativa de aquellas, que impiden que los PE para los caracteres MDF, DE, CDV, CEM, FINO15, FDEC, FDEH, LMECHA, FPFT y CRV de la fibra del vellón de alpacas de la zona del estudio sean determinados de la manera más recomendable y tal vez más confiables

Otros investigadores determinan PE por diversos procedimientos metodológicos para diferentes características y en diferentes especies de animales domésticos. Así, El-Arian (2005) construye 26 índices de selección para características de leche en ganado vacuno Holstein; Gwaza et al. (2018) determinan PE para algunas características de la producción de leche en ganado Holstein y cruces; Kabirul y Mazumder (2011) lo hacen para caracteres de producción de leche en ganado Holstein y sus cruces; en tanto que Quinton et al. (2006) lo emplean para características productivas en una población de cerdos. No obs- 
tante, todos usan la función beneficio, que toma en consideración la ganancia económica que produce cada rasgo biológico al ser mejorado en una unidad, asumiendo que los otros rasgos permanecen constantes (Urioste et al., 1998).

De manera similar, existen investigaciones que recurren a los modelos bioeconómicos para determinar PE. Vargas et al. (2002) utilizan un modelo que combina la performance del potencial genético, estrategias de consumo de alimento, una saca optima y políticas de inseminación en ganado Holstein; Ivanovic et al. (2014) refieren la determinación de PE para características productivas lácteas en la mejora de la ganadería de leche en Serbia, en tanto que Miesenberger et al. (1998) lo utilizan en características de fertilidad y reproducción asumiendo una función beneficio con un modelo hato antes y después del cambio genético en vacunos.

Algunos investigadores utilizaron el método del recíproco de la DE fenotípica $\left(1 / \sigma_{\mathrm{p}}\right)$ para determinar PE relativos para la construcción de índices de selección. Por ejemplo, Atil (2006) los determina para los caracteres rendimiento de leche ajustado a 305 días, periodo de lactación y edad al primer parto en ganado Holstein; Moniem y Salah (2009) para caracteres de rendimiento total de leche, periodo de lactación, peso al nacimiento y peso al destete en búfalos; Chin et al. (2015) para diversos caracteres productivos a objeto de mejorar la producción de carne; en tanto que Apaza y Quispe (2014) refieren el PE para características de peso de manifestación temprana en llamas.

La tecnología de regresión también permite la estimación de los valores o pesos económicos (PE). Desde esta perspectiva, los pesos económicos son definidos como la regresión parcial de $H$ sobre $g$ (James, 1982); valores que pueden modificarse cuando algunos de los caracteres no son considerados en el modelo; sin embargo, estos constituyen una aproximación de los verdaderos pesos económicos; aunque procedimentalmente presentan algunos problemas como la precisión de la rentabilidad o el ingreso total; asimismo los sistemas de manejo ganadero usuales no necesariamente son los mismos en todos los hatos, el número de animales por hato es diferente, y otros aspectos más, que hacen que la estructura de variancias y covariancias sea diferente de una población a otra. Además, los estimados de PE a través de la regresión múltiple son dependientes de las características incluidas en el análisis. Lembeye et al., (2014) utiliza el procedimiento de la regresión para determinar PE en la tasa de crecimiento y peso al nacer en ovinos Suffolk Down en condiciones extensivas de la zona central de Chile.

Los valores de PE hallados en las investigaciones referidas no son factibles de ser contrastados con los obtenidos en el presente estudio por pertenecer a caracteres diferentes, corresponden a otras especies domésticas y sistemas de producción y utilizan otros procedimientos metodológicos. Los resultados obtenidos en el presente estudio, por ambos procesos metodológicos, hacen referencia a la especie alpaca y son únicos, cuyos valores a pesar de ser relativos, se adicionan excepcionalmente al cúmulo de conocimientos en este entorno, para las características relacionadas a la fibra de alpacas, en un sistema de explotación propia de esta especie.

\section{Conclusiones}

- Se han determinado pesos económicos relativos para 10 caracteres relacionados a la finura de la fibra de alpacas Huacaya mediante dos procedimientos metodológicos, al objeto de estructurar un índice para el agregado genético total. Estos en su totalidad no necesariamente constituyen criterio de selección, en cuyo caso estos pesos económicos pueden variar. 
- Las magnitudes de los PE, en cada caso, representan el cambio en el valor de cría del agregado genético total, debido a un incremento independiente de una unidad de mejora genética en ese carácter. Excepto en aquellos cuyo valor se encuentra precedido del signo negativo cuando son obtenidos por el método de regresión, que implica una disminución en la performance de dicho carácter, beneficiosa para algunos caracteres, circunstancia paradójica producto del método usado.

\section{Literatura Citada}

1. ALT-PNUD. 2001. Informe final: Determinar la capacidad de carga de los bofedales para la alpaca en el ámbito peruano. Proyecto PER/98 G-32. Puno Perú.

2. Apaza E, Quispe JE. 2014. Índices de selección genética para caracteres de pesos al nacimiento, destete y ganancia de peso en llamas (Lama glama, linnaeus, 1.758). Rev Investig Altoandin 2014: 16: 21-32. doi: 10.18271/ria.2014.31

3. Atil H. 2006. A comparison of different selection indices for genetic improvement for milk traits in Holstein Friesian cattle in turkey by using one standard deviation as relative economic weight. Pak J Biol Sci 9: 285-288. doi:10.1080/ 09712119.2005.9706553

4. Bobner C. 1994. Schatzung wirtschaftlicher Gewichte fur sekundare Leistunbsmerkmale bei schweizrischeb zweinutzungsrrindem unter anwendum der dynamischen optimierum. PhD Thesis. Switzerlan: Federal Technical University Zurich. 127 p.

5. Bourdon RM. 1998. Shortcomings of current genetic evaluation systems. In J Anim Sci 76: 2308-2323.

6. Brascamp EW. 1978. Methods on economic optimization of animal breeding plans. Report B 134, Research Institute for Animal Production «Schoonoord», The Netherlands. $177 \mathrm{p}$.

7. Charfeddine N. 2000. Economic aspects of defining breeding objectives in selection programmes. In: Gabiña D (ed). Analysis and definition of the objectives in genetic improvement programmes in sheep and goats. An economic approach to increase their profitability. Zaragoza, España: CIHEAM. p 9-17.

8. Chen J, Wang Y, Zhang Y, Sun D, Zhang Y. 2009. Estimation of economic values for production and functional traits in Chinese Holstein. J Anim Vet Adv 8: 2125-2132.

9. Chin RC, Magaña JG, Segura JC, Rafael DR, Estrada RJ. 2015. Índices de selección para el mejoramiento productivo de bovinos suizo europeo en México. Ecosistemas y Recursos Agropecuarios 3: 1-9.

10. Duangjinda M. 2007. SAS/IML. For best linear unbiased predicition. Thailand: Khon Kaen University. [Internet]. Available in: https://docplayer.net/ 101841418-Sas-iml-for-best-linearunbiased-prediction.html

11. El-Arian MN. 2005. Selection indices for Friesian cows using two methods of calculating relative economic values for some important productive and reproductive traits. J Agric Sci Mansoura Univ Egypt 30: 7285-7296.

12. Elsen JM, Bibe B, Landais E, Ricorddeau G. 1986. Twenty remarks on economic evaluation of selection goals. In. Proc $3^{\text {rd }}$ World Congresses on Genetics Applied to Livestock Production. Lincoln, USA.

13. Elvira M. 2014. Presentación del instrumento de medición de finura OFDA 2000: Uso y aplicaciones. https://docplayer.es/44479138-Presentacion-del-instrumento-de-medicion-de-finuraofda.html

14. Falconer DS, Mackay TFC. 1996. Introducción a la genética cuantitativa. Zaragoza, España: Acribia. 500 p. 
15. Fernández M, Alenda R, 1999. Pesos económicos en vacunos de carne de la raza avileña negra ibérica para la aplicación a un programa de mejora. [Internet]. Disponible en: https:// acteon.webs.upv.es/CONGRESOS/ AIDA\%201999/mfernandez.htm

16. Gibson, JP, Graham N, Burnside EB. 1992. Selection index for production traits of Canadian dairy sires. Can J Anim Sci 72: 477-491.

17. Groen A. 1989. Cattle breeding goals and production circumstances. $\mathrm{PhD}$ Thesis. The Netherlands: Wageningen Agricultural University. $167 \mathrm{p}$.

18. Groen AF, Steine T, Colleau JJ, Pedersen J, Pribyl J, Reinsch N. 1997. Economic values in dairy cattle breeding, with special reference to functional traits. Livest Prod Sci 49:1-21. doi: 10.1016/S0301-6226(97)00041-9

19. Gwaza DS, Mohammed G, Addas PA. 2018. Derivation of economic eights of milk components as selection criteria for genetic improvement of dairy animals. J Res Rep Genet 2: 7-13.

20. Harris BL, Freeman AE. 1993. Economic weights for milk yield traits and herd life under various economic conditions and production quotas. J Dairy Sci 76: 868-879. doi: 10.3168/jds.S00220302(93)77413-5

21. INDECOPI. 2014. NTP 231.302:2014. Fibra de alpaca clasificada. Definiciones, clasificación por grupos de calidades, requisitos y rotulados. $2^{\circ}$ Edición. Comisión de Normalización y de Fiscalización de Barreras Comerciales no Arancelarias. Normas legales. Boletín del Diario Oficial El Peruano. 07/09/2014

22. Ivanovic S, Stanojevic D, Nastic L, Jelocnic M. 2014. Determination of economic selection index coefficients for dairy caws. Ekonomika Poljoprivrede 61: 861-875. doi: 10.5937/ekoPolj1404861I

23. James JW. 1982. Construction, uses, and problems of multitrait selection indices. In: Proc $2^{\text {nd }}$ World Congresses on Genetics Applied to Livestock Production. Madrid, Spain.
24. Kahi AK, Nitter G. 2004. Developing breeding schemes for pasture based dairy production systems in Kenya. Derivation of values using profit functions. Livest Prod Sci 88: 161-177. doi: 10.1016/ j.livprodsci.2003.10.008

25. Kabirul IK, Mazumder J. 2011. Economic selection index using different milk production traits of Holstein and its crossbreds. Turk J Vet Anim Sci 35: 255 261. doi:10.3906/vet-1005-34

26. Kluyts JF, Neser FWC, Bradfield MJ. 2007. Derivation of economic values for the Simmentaler breed in South Africa. S Afr J Anim Sci 37:121. Doi: 10.13140/ 2.1.4061.2482

27. Krupová Z, Oravcová M, Krupa E, Peskovicová D. 2008. Methods for calculating economic weights of important traits in sheep. Czech J Anim Sci 41: 24-29.

28. Legarra A, Ramón M, Ugarte E, Pérez MD. 2007. Economic weights of fertility, prolificacy, milk yield and longevity in dairy sheep. Animal 1: 193203. doi: $10.1017 / \mathrm{S} 1751731107657814$

29. Lembeye F, Castellaro G, Magofke JC, Uribe H. 2014. Comparación de criterios de selección para ovinos Suffolk Down manejados en condiciones extensivas en la zona central de Chile. Arch Med Vet 46: 239-246.

30. MacNieil MD, Nungent RA, Snelling WM. 1997. Breeding for profit: an introduction to selection index concepts. University of Nebraska, Lincoln. [Internet]. Available in: http://digitalcommons.unl.edu/rangebeefcowsymp/142

31. Miesenberger J, Sölkner J, Essl A. 1998. Economic weights for fertility and reproduction traits relative to other traits and effects of including functional traits into a total merit index, In: Proc International Workshop on Genetic Improvement of Functional Traits in Cattle. Grub, Germany.

32. Moniem KA, Salah A. 2009. A comparison between different selection indices for some productive traits on Egyptian buffaloes. Arch Tierzucht 5: 476-484. doi: 10.5194/aab-52-476-2009 
33. Mueller JP. 1985. Implementación de planes de mejoramiento genético en ovinos. I. Objetivos de mejoramiento y criterios de selección. INTA Bariloche. Comunicación Técnica $\mathrm{N}^{\circ} 6$.

34. Parna E, Saveli O, Kaart T. 2002. Economic weights for production and functional traits of Estonian Holstein population. In: Proc $7^{\text {th }}$ World Congress on Genetics Applied to Livestock Production. Montpellier, France.

35. Pearson RE, Miller RH. 1981. Economic definition of total performance, breeding goals, and breeding values for dairy cattle. J Dairy Sci 64: 857-869. doi: 10.3168/jds.S0022-0302(81)82659-8

36. Piedrafita AJ. 1995. Predicción de la respuesta a la selección. En: Buxadè $C$ (ed). Genética, patología, higiene y residuos animales. Madrid, España: Mundi Prensa. p139-150.

37. Ponzoni R, Newman S. 1989. Developing breeding objectives for Australian beef cattle production. Anim Prod 49: 35 47. doi: $10.1017 / \mathrm{S} 0003356100004232$

38. Quinton VM, Wilton JW, Robinson JA, Mathur PK. 2006. Economic weights for sow productivity traits in nucleus pig population. Livest Sci 99: 69-77. doi: 10.1016/j.livsci.2005.06.002

39. Quispe JE, Apaza ZE, Quispe DM, Morocco NL. 2016. De vuelta a la alpaca. La producción primaria en una perspectiva empresarial y competitiva. Perú: Univ. Nacional del Altiplano. $450 \mathrm{p}$.

40. Rabelo Da Fonseca GE, Pruzzo L, Maizon DO, Mirande SL. 2000. Definición del objetivo económico de selección para un sistema de producción porcina en Argentina. Investigación agraria. Producción y Sanidad Animales 15: 5-19.
41. Ramón M, Legarra A, Pérez-Guzmán MD, Ugarte E. 2005. Obtención de pesos económicos para selección por rentabilidad. Investigación agraria. Producción y Sanidad Animales 15: 5-19.

42. Rewe TO, Indetie D, Ojango JM, Kahi $A K$. 2006. Economic values for productions and functional traits and assessment of their influence on genetic improvement in the Boran cattle in Kenya. J Anim Breed Genet 123: 23-36. doi: 10.1111/ j.1439-0388.2006.00558.x

43. Romero PJM. 2000. Métodos de selección para caracteres múltiples. Tesis de Estadístico. México: Univ. Autónoma Chapingo.

44. Saveli O, Parna E, Dewi IA. 2003. Derivation of economic values of beef production traits of Estonian Holstein cattle. In: EFITA Conference. Debrecen, Hungary.

45. Urioste JI, Ponzoni RW, Aguirrezabala M, Rovere G, Saavedra $D$. 1998. Breeding objectives for pasturefed Uruguayan beef cattle. J Anim Breed Genet 115: 357-373. doi: 10.1111/j.14390388.1998.tb00357.x

46. Vargas B, Groen AF, Herrero $M$, Van Arendonk J AM. 2002. Economic values for production and functional traits in Holstein cattle of Costa Rica. Livest Prod Sci 75: 101-116

47. Weigel DJ, Cassell BG, Hoeschelle I, Pearson RE. 1995. Multiple trait prediction of transmitting abilities for herd life and estimation of economic weights using relative net income adjusted for opportunity cost. J Dairy Sci 78: 639-647. doi: 10.3168/jds.S0022-0302(95)76675-9

48. Yáñez L, Aranguren-Méndez J, Villasmil-Ontiveros Y, Rojas N. 2006. Modelo bio-económico de simulación para orientar la definición del objetivo de Selección en el sistema de doble propósito. Rev Cient-Fac Cien Vet 4: 381-392. 